\title{
METILFENIDATO: REPERCUSSÕES SISTÊMICAS DO USO POR ESTUDANTES
}

\author{
Categoria: SAÚDE COLETIVA/ EPIDEMIOLOGIA \\ CENTRO UNIVERSITÁRIO SÃO CAMILO
}

\author{
Constantino, G. $\mathbf{A}^{1}$; \\ Oliveira, G.E.S.'; \\ Andako, G.K.'; \\ Lee, K.H.R. ${ }^{1}$; \\ Alcoforado, L.V.'; \\ Saramago, M.S.'; \\ Manso, M.E.G. ${ }^{2}$
}

\begin{abstract}
'ACADÊMICOS DO CENTRO UNIVERSITÁRIO SÃO CAMILO 2PROFESORA TITULAR DA SÃO CAMILO, MÉDICA, BACHAREL EM DIREITO, MESTRE EM GERONTOLOGIA SOCIAL, DOUTORADO EM CIÊNCIAS SOCIAIS E PÓS DOUTORADO EM GERONTOLOGIA SOCIAL E PSICOGERONTOLOGIA
\end{abstract}

\section{São Paulo}

2019

R. Teodoro de Beaurepaire, 197 - Vila Dom Pedro I, São Paulo - SP, CEP: 04279-030

(15) 997268770 e-mail: constalm.gabriela@gmail.com 


\title{
METILFENIDATO: REPERCUSSÕES SISTÊMICAS DO USO POR ESTUDANTES
}

\section{Categoria: SAÚDE COLETIVA/ EPIDEMIOLOGIA}

\author{
DESCRITORES: Metilfenidato; saúde
}




\section{METILFENIDATO: REPERCUSSÕES SISTÊMICAS DO USO POR ESTUDANTES}

AUTORES : Constantino, G. A; Oliveira, G.E.S.; Andako, G.K.; Lee, K.H.R.; Alcoforado, L.V.; Saramago, M.S.

ORIENTADORA: Manso, M.E.G.

INSTITUIÇÃO: Centro Universitário São Camilo

\section{RESUMO :}

INTRODUÇÃO:Com a constante e crescente pressão acadêmica presente na realidade de vestibulandos e universitários, muitos recorrem ao uso de psicoestimulantes como o metilfenidato. O metilfenidato, que tem a Ritalina ${ }^{\circledR}$ como forma comercial mais conhecida, é recomendado, principalmente, para o tratamento de transtorno de déficit de atenção com hiperatividade (TDAH), mas seu uso não prescrito traz repercussões sistêmicas graves que são negligenciadas.

OBJETIVOS: Esta revisão propõe analisar a literatura acerca do uso sem prescrição de metilfenidato em adolescentes e adultos saudáveis, a fim de entender seu impacto na saúde.

METODOLOGIA: Pesquisa do tipo revisão bibliográfica sobre a repercussão sistêmica do uso de metilfenidato por estudantes utilizando BVS e Pubmed, tendo como descritores encontrados no DeCS: "saúde" e "metilfenidato". Foram incluídos artigos de revistas indexadas com textos completos disponibilizados online, publicados nas línguas portuguesa e inglesa, no período de 2014 a 2018; excluídos textos que abordavam estudos com pessoas fora da faixa etária determinada e textos que possuíam estudos com pessoas portadoras de TDAH. Foram acrescentados ainda 2 artigos provenientes de revista não indexadas devido a escassez de informações complementares.

RESULTADOS: Apesar da obtenção de pequena quantidade de estudos voltados ao tema em questão, foram constatados impactos desse fármaco no organismo, principalmente no sistema nervoso central e no sistema cardiovascular. Quanto ao SNC, evidenciaram-se alterações nos níveis de neurotransmissores e no metabolismo cerebral acarretando, também, mudanças comportamentais. Já no sistema cardiovascular, a análise dos estudos apresentou informações divergentes quanto a atuação exata do fármaco, porém sem negar que há alteração nesse sistema com o uso do psicoativo.

CONCLUSÃO: Após a análise dos artigos selecionados, foram constatadas que, devido ao uso dessa substância, ocorrem alterações no cerebelo e no cérebro, as quais intensificam a memória e concentração. Além disso, verificou-se efeitos colaterais como ansiedade, elevação da pressão arterial e da frequência cardíaca e dor abdominal. Apesar dos resultados encontrados, há uma carência em artigos publicados e assim, faz-se necessário mais estudos acerca do tema.

DESCRITORES: metilfenidato,saúde. 


\title{
METILFENIDATO: REPERCUSSÕES SISTÊMICAS DO USO POR ESTUDANTES
}

\section{RESUMO}

O metilfenidato é um princípio ativo de fármacos estimulantes do sistema nervoso central. Seu uso foi amplamente difundido entre vestibulandos e estudantes universitários com o objetivo de aprimorar o rendimento acadêmico, mas suas repercussões sistêmicas são muitas vezes negligenciadas. Esta revisão propõe analisar a literatura acerca do uso sem prescrição do fármaco em adolescentes e adultos saudáveis, a fim de entender seu impacto na saúde. Observou-se efeitos de curto e longo prazo, destacando principalmente as alterações no sistema nervoso e cardíaco. Com isso entende-se que, por se tratar de um fármaco potente, seu uso sem prescrição não é recomendado.

\section{PALAVRAS CHAVE: metilfenidato, saúde}

\begin{abstract}
Methylphenidate is a pharmaceutical active principle that acts in the central nervous system. Its use has been widespread among college and high school students, aiming at academic efficiency improvement. However, the drug's systemic repercussions are often neglected by these students. This review plans to analyze literature about methylphenidate use without medical prescription in teenagers and healthy adults, in order to understand its health impacts. It is noted that there are short and long-term effects, highlighting changes in nervous and cardiac system. For this reason, it is understood that the misuse of this powerful medication is not recommended.
\end{abstract}

KEY WORDS: methylphenidate, health

\section{INTRODUÇÃO}

Os estudantes universitários vivem uma realidade marcada por constante pressão social. A partir do ensino médio até a conclusão do ensino superior, exige-se dos alunos um rendimento sobre-humano (BARROS; ORTEGA, 2010).

Percebe-se que, desde muito cedo, os adolescentes convivem com as pressões familiares e as incertezas acerca dos caminhos que desejam seguir, e são obrigados a escolher 
muito precocemente seu futuro profissional. Ademais, existe uma enorme competitividade no ingresso às universidades brasileiras, o que torna o processo até a aprovação bastante difícil e desgastante. Ao conquistar a sua vaga, os estudantes chegam às faculdades cheios de expectativas e ideais. No entanto, o cenário encontrado por eles muitas vezes não condiz com as expectativas. Não atingir seus objetivos acadêmicos, alterações no seu cotidiano (mudança de cidade, casa, grade horária etc), privação de sono, carga excessiva de estudos, falta de tempo, dificuldade de conciliar atividades de lazer, cansaço físico e psicológico são alguns dos fatores que sobrecarregam os estudantes (MILLAN et al. apud AZEVEDO; MOREIRA; SILVA; TERTULINO; VILAR, 2006).

Outro ponto a se destacar é a relação entre cumprir a exigência social em vários aspectos, entre eles de excelente performance, e a aceitação do grupo social à qual o jovem está inserido (BARROS; ORTEGA, 2010). Essa associação gera uma competitividade exacerbada, e as possíveis frustrações advindas das quebras de expectativa, do não cumprimento de imposições sociais e do desapontamento em relação ao desempenho acadêmico, levam à um sentimento de fracasso e angústia. Assim, para cumprir essas exigências desumanas impostas pela sociedade, os estudantes passaram a adotar a prática da automedicação com fármacos que melhoram seu rendimento acadêmico (BARROS; ORTEGA, 2010).

A automedicação é a prática de ingerir substâncias de ação medicamentosa sem o aconselhamento e/ou acompanhamento de um profissional de saúde qualificado. É um procedimento caracterizado pela iniciativa de um doente ou de seu responsável, em obter ou produzir e utilizar um produto para o tratamento de doenças ou alívio de sintomas (PAULO \& ZANINI, 1988 apud ARRAIS, 1997; CASTRO, 2006). O aumento da expectativa de vida da população e o consequente crescimento da incidência de doenças crônicas, somados ao surgimento de novas doenças transmissíveis e o reaparecimento de antigas, mais casos de transtornos de humor e de doenças resultantes da poluição ambiental são fatores que estimulam a automedicação (ARRAIS, 1997).

No Brasil, a automedicação é justificada, na maioria dos casos, pela má qualidade e demora no atendimento do sistema público de saúde, associados ao fácil acesso à farmácias (NAVES, 2010). Os medicamentos mais consumidos pelos brasileiros, de acordo com o primeiro nível da classificação ATC (Anatomical Therapeutic Chemical), são os de ação no sistema nervoso central, músculo-esquelético, trato alimentar e metabolismo, sistema 
respiratório, sistema geniturinário e hormônios sexuais, anti-infecciosos para uso sistêmico e outros (ARRAIS, 1997).

Entre os medicamentos para o sistema nervoso central apontados como os mais consumidos sem prescrição médica, encontra-se o metilfenidato, alvo desta revisão, sendo o seu uso sem orientação de um profissional de saúde motivado pelos seus efeitos potencializadores do desempenho cognitivo (CARNEIRO, 2013). Devido à essa ação, muitos estudantes buscam tal droga por conta da alta cobrança das universidades em suas bases curriculares, não levando em consideração os diversos efeitos colaterais na fisiologia do organismo humano (CARNEIRO, 2013).

O Metilfenidato é um medicamento com estrutura análoga à anfetamina, sendo a Ritalina ${ }^{\circledR}$ a forma comercial mais conhecida. Essa substância é considerada um estimulante do Sistema Nervoso Central, aumentando os níveis de catecolaminas no meio extracelular, a exemplo dos neurotransmissores norepinefrina (noradrenalina) e dopamina (VOLKOW et al., 2001; BERRIDGE et al., 2006; SPENCER et al., 2015 apud CRUZ, 2011 ). Além disso, ela estimula os receptores alfa e beta adrenérgicos (PASTURA, 2004) . O uso desse fármaco é recomendado principalmente para o tratamento de transtorno do déficit de atenção com hiperatividade (TDAH). Entretanto, devido aos seus efeitos, nos últimos tempos ele tem sido utilizado por pessoas saudáveis sem prescrição médica.

O objetivo deste trabalho é revisar a literatura acerca do uso não prescrito de metilfenidato e as repercussões sistêmicas causadas no organismo de vestibulandos e universitários.

\section{METODOLOGIA}

Esse estudo corresponde à pesquisa do tipo revisão bibliográfica.

A coleta de dados foi realizada utilizando-se as palavras-chaves encontradas no DeCS: "saúde" e "metilfenidato". Foram incluídos artigos com textos completos disponibilizados online, publicados nas línguas portuguesa e inglesa, no período de 2014 a 2018. Excluídos textos que abordavam estudos com pessoas fora da faixa etária do estudo (15 a 25 anos) e textos que abordavam estudos em pessoas portadoras de TDAH.

A investigação para a identificação dos artigos ocorreu em cinco etapas: inicialmente foram identificados 2869 trabalhos utilizando os descritores "saúde" e "metilfenidato" nas 
bases de dados BVS e PubMed; em seguida foram excluídos artigos não disponíveis, trabalhos que não se encaixavam na delimitação do tema e artigos que após a leitura do título e resumo não se encaixavam no tema proposto por esta presente revisão. Na figura, apresenta-se graficamente a seleção dos artigos para a revisão.

Conclui-se com 7 trabalhos dos quais dois, após a leitura na íntegra, foram excluídos. Devido à dificuldade em encontrar artigos em português acerca do tema desenvolvido, foram acrescentados ainda, 2 artigos provenientes de revista não indexada.

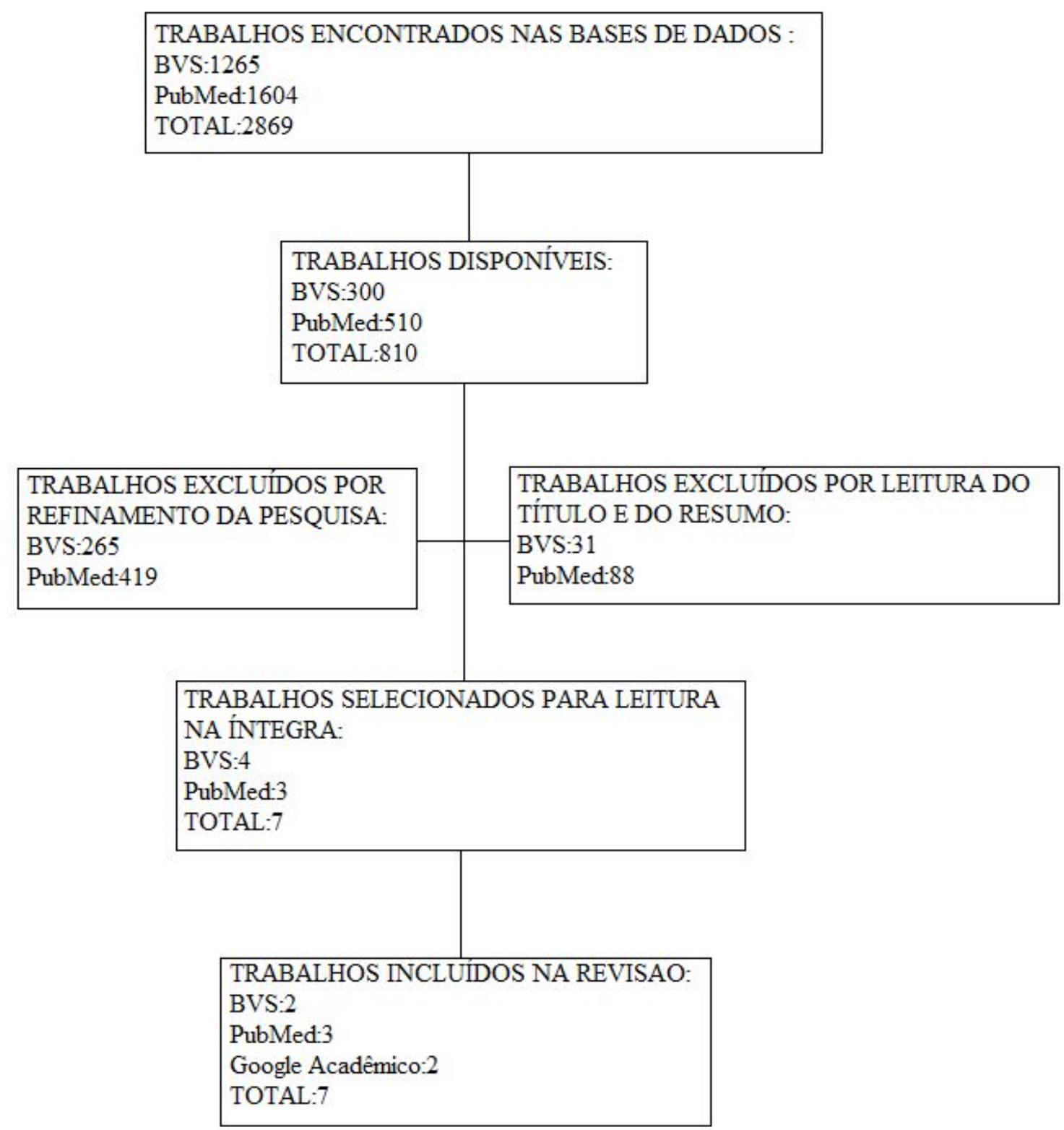

Figura 1: Representação gráfica das etapas realizadas para a revisão 


\section{RESULTADOS}

No quadro 1 são apresentados os trabalhos selecionados e as conclusões inferidas pela leitura de cada um.

Quadro 1: Resultados

\begin{tabular}{|c|c|c|c|}
\hline AUTORES & TÍTULO & MÉTODO & CONCLUSÃO \\
\hline $\begin{array}{l}\text { Ahmad Ali } \\
\text { Eslami et al }\end{array}$ & $\begin{array}{c}\text { Intention and } \\
\text { Willingness in } \\
\text { Understanding } \\
\text { Ritalin Misuse } \\
\text { Among Iranian } \\
\text { Medical College } \\
\text { Students: A } \\
\text { Cross-Sectional } \\
\text { Study }\end{array}$ & Qualitativa & $\begin{array}{l}\text { É possível desenvolver um } \\
\text { projeto de intervenção com o } \\
\text { objetivo de demonstrar que } \\
\text { metas acadêmicas podem ser } \\
\text { atingidas sem o uso inadequado } \\
\text { de Ritalina. }\end{array}$ \\
\hline $\begin{array}{c}\text { Ana Carolina } \\
\text { Pereira da Silva } \\
\text { et al }\end{array}$ & $\begin{array}{l}\text { A explosão do } \\
\text { consumo de ritalina }\end{array}$ & Qualitativa & $\begin{array}{l}\text { Menciona os benefícios, da } \\
\text { melhora no desempenho, no } \\
\text { transtorno de déficit de atenção, } \\
\text { etc. Porém, quase não há } \\
\text { menção acerca dos defeitos } \\
\text { psíquicos marcantes causadas } \\
\text { pelo uso contínuo desse } \\
\text { fármaco }\end{array}$ \\
\hline
\end{tabular}




\begin{tabular}{|c|c|c|c|}
\hline $\begin{array}{l}\text { Ernst, Monique } \\
\text { et al }\end{array}$ & $\begin{array}{c}\text { The effects of } \\
\text { methylphenidate } \\
\text { and propranolol on } \\
\text { the interplay } \\
\text { between } \\
\text { induced-anxiety and } \\
\text { working memory }\end{array}$ & $\begin{array}{l}\text { Quantitativ } \\
\text { a }\end{array}$ & $\begin{array}{l}\text { Melhoras na cognição, } \\
\text { relacionadas ao uso de } \\
\text { metilfenidato, permitem que } \\
\text { ansiedade possa ser processada. }\end{array}$ \\
\hline $\begin{array}{c}\text { Marcio } \\
\text { Henrique de } \\
\text { Moura }\end{array}$ & $\begin{array}{l}\text { As consequências do } \\
\text { uso prolongado e não } \\
\text { terapêutico do } \\
\text { metilfenidato }\end{array}$ & Qualitativa & $\begin{array}{l}\text { O uso abusivo e sem prescrição } \\
\text { do metilfenidato, } \\
\text { principalmente pelo público } \\
\text { acadêmico, gera diversas } \\
\text { consequências à saúde do } \\
\text { paciente, tanto em curto prazo } \\
\text { quanto a longo prazo. }\end{array}$ \\
\hline $\begin{array}{c}\text { Monica } \\
\text { Rosenberg et al }\end{array}$ & $\begin{array}{c}\text { Methylphenidate } \\
\text { Modulates } \\
\text { Functional Network } \\
\text { Connectivity to } \\
\text { Enhance Attention }\end{array}$ & $\begin{array}{l}\text { Quantitativ } \\
\text { a }\end{array}$ & $\begin{array}{l}\text { Adultos saudáveis sob efeito de } \\
\text { metilfenidato apresentaram } \\
\text { conexões cerebrais condizentes } \\
\text { com fortes habilidades de } \\
\text { atenção. O estudo apresenta } \\
\text { limitações, como o não uso de } \\
\text { placebo no grupo controle, } \\
\text { entretanto os pesquisadores } \\
\text { acreditam que elas não afetam } \\
\text { as conclusões. }\end{array}$ \\
\hline
\end{tabular}




\begin{tabular}{|c|c|c|c|}
\hline $\begin{array}{l}\text { Peter Manza et } \\
\text { al }\end{array}$ & $\begin{array}{l}\text { The effects of } \\
\text { methylphenidate on } \\
\text { cerebral responses } \\
\text { to conflict } \\
\text { anticipation and } \\
\text { unsigned prediction } \\
\text { error in a } \\
\text { stop-signal task }\end{array}$ & $\begin{array}{l}\text { Quantitativ } \\
\text { a }\end{array}$ & $\begin{array}{l}\text { O metilfenidato aumenta a } \\
\text { ativação do núcleo caudado na } \\
\text { antecipação de conflitos e reduz } \\
\text { a ativação cerebral na previsão } \\
\text { de eventos adversos no controle } \\
\text { de impulso. Entretanto, o } \\
\text { estudo não teve um controle } \\
\text { com placebo para os indivíduos } \\
\text { que receberam metilfenidato. } \\
\text { Devido à isso, podem haver } \\
\text { variações nos resultados. }\end{array}$ \\
\hline $\begin{array}{l}\text { Westover AN et } \\
\text { al }\end{array}$ & $\begin{array}{l}\text { Impact of Stimulant } \\
\text { Medication Use on } \\
\text { Heart Rate and } \\
\text { Systolic Blood } \\
\text { Pressure During } \\
\text { Submaximal } \\
\text { Exercise Treadmill } \\
\text { Testing in } \\
\text { Adolescents. }\end{array}$ & $\begin{array}{l}\text { Quantitativ } \\
\text { a }\end{array}$ & $\begin{array}{l}\text { Observou-se uma tendência de } \\
\text { diminuição da frequência } \\
\text { cardíaca durante exercícios de } \\
\text { alta intensidade em usuários de } \\
\text { metilfenidato, o que pode } \\
\text { evidenciar adaptações crônicas } \\
\text { nos usuários desses } \\
\text { medicamentos. }\end{array}$ \\
\hline
\end{tabular}

\section{DISCUSSÃO}

O metilfenidato é um princípio ativo de fármacos estimulantes do Sistema Nervoso Central, sendo classificado como um psicoanaléptico. Por ser um medicamento muito eficiente, os sinais comportamentais e de tolerância surgem rapidamente, o que aumenta o risco de abuso e de dependência. Devido a isso, a droga é de uso controlado (SADOCK et al., apud ESLAMI et al., 2014 ) .

O modo de ação dessa substância no organismo humano não é totalmente conhecido mas acredita-se que ele estimula a região do Córtex Cerebral e, presumivelmente o sistema de 
excitação reticular (NOVARTIS, apud SILVA, 2017). Apesar desse mecanismo não ser preciso, seu uso não prescrito é altamente disseminado entre estudantes, os quais alegam melhora no desempenho acadêmico, mas mostram falta de atenção para os efeitos colaterais (ESLAMI et al., 2014).

Os efeitos colaterais podem ser divididos entre curto e longo prazo. Dentre os de curto prazo, os que mais predominam são a diminuição do apetite, insônia, cefaleia, dor abdominal, labilidade emocional e ansiedade. Já os de longo prazo, destacam-se dependência, efeitos cardiovasculares e possível redução da estatura. (BARKLEY et al; LLANA, CRISMO, apud MOURA, 2017)

A ansiedade está relacionada ao bloqueio dos transportadores de norepinefrina e dopamina, aumentando os níveis desses neurotransmissores na fenda sináptica (ROSEMBERG et al., 2016). Essa informação levou alguns autores a concluírem que há aumento das ondas de atividade cerebral (HIRATA et al.; KIYATKIN, REBEC, apud MANZA et al., 2016), pois o metilfenidato pode reduzir a energia necessária à conclusão de atividades cognitivas através da diminuição do metabolismo de glicose no cérebro, tornando-o mais eficiente. (COOLS, D’ESPOSITO, apud MANZA et al., 2016).Outros estudos consideram que a melhora na eficiência cognitiva adquirida com o uso de metilfenidato permite que a ansiedade seja processada e expressada (ERNST)

Ao comparar a administração de metilfenidato em adultos saudáveis e em pessoas com deficiência de dopamina (TDAH), observou-se que enquanto o fármaco resulta em um excesso de dopamina em pessoas saudáveis (COOLS, 2006, apud MANZA et al., 2016), ela normaliza a atividade cerebral na resposta de inibição em indivíduos com a deficiência (VAIDYA et al., 1998, apud MANZA et al., 2016). Portadores de TDAH apresentam uma performance insuficiente em tarefas que exigem a antecipação de conflitos e demandas cognitivas. Visto isso, a administração da droga aperfeiçoa o desempenho dessas atividades devido às mudanças na função da catecolamina do núcleo caudado (MANZA et al., 2016).

Apesar do fármaco melhorar a memória e controles inibitórios (ROSENBERG et al., 2016), o metilfenidato acarreta diversas mudanças no cérebro de indivíduos saudáveis, modificando e ativando diversas regiões do órgão. Na realização de tarefas, por exemplo, usuários do psicoestimulante apresentam aumento das conexões entre as regiões occipital e motora, e do sistema límbico e pré-frontal. Já no descanso, há o aumento das conexões entre as regiões do sistema límbico e motora, entre o córtex parietal e o córtex occipital, entre o 
córtex occipital e o córtex insular, entre o lobo temporal e regiões do tronco encefálico. Ao contrário disso, pessoas que não fazem o uso do medicamento apresentam o aumento, durante a realização de tarefas, das conexões que agem no descanso em indivíduos medicados (ROSENBERG et al., 2016). Em jovens saudáveis, a administração de metilfenidato está associada à amplificação das respostas cerebrais para a antecipação de conflitos e para a diminuição da previsão de eventos adversos. Logo, há maior ativação no núcleo caudado bilateral e no tálamo dorsal em usuários de metilfenidato (MANZA et al., 2016).

A respeito da repercussão no sistema cardiovascular há divergências. Alguns estudos apontam que ela é pontual e transitória, podendo-se observar uma elevação na pressão arterial sistólica de aproximadamente $2 \mathrm{mmHg}$ e na frequência cardíaca de 5.7 batimentos por minuto logo após o uso do metilfenidato (FINDLING et al., apud at MOURA, 2017 e STOWE et al., SAMUELS et al., apud at WESTOVER et al., 2016).

Em contraposição, durante testes de exercício com esforço submáximo, os usuários de metilfenidato, quando comparados aos não-usuários, apresentaram menor pico de frequência cardíaca e tempo de recuperação da FC alterado, o que sugere sub-sensibilidade dos receptores beta cardíacos; eles também apresentaram menor pressão arterial sistólica durante o aquecimento. Observou-se ainda que a teoria que associa medicamentos estimulantes, incluindo o metilfenidato, com a "síndrome do estresse sistêmico crônico" pode estar relacionado com esses resultados . (WESTOVER et al., 2016)

\section{CONCLUSÃO}

Nos artigos selecionados para essa revisão foram constatados efeitos do metilfenidato dentro e fora do sistema nervoso central observando ações no cerebelo e nas regiões motoras, parietal, temporal e occipital, o que intensifica a memória e concentração. Todavia, foram relatados outros impactos no organismo como ansiedade, labilidade emocional, dependência, insônia, dor abdominal e consequente diminuição de apetite, cefaléia e possível redução da estatura. Além disso, no sistema cardiovascular foram evidenciadas elevação da pressão arterial e elevação da frequência cardíaca. Dessa forma, infere-se que o uso sem prescrição de metilfenidato é altamente desaconselhável.

Apesar dos resultados encontrados, faz-se necessário mais estudos sobre o modo de ação do metilfenidato, tendo em vista a carência de artigos publicados acerca do tema e a 
insuficiência de suas conclusões. Com o aprofundamento no assunto, será possível um melhor entendimento da repercussão sistêmica desse medicamento, o que deverá ser disseminado à população leiga visando diminuir o uso inadequado desse psicoestimulante.

\section{REFERÊNCIAS}

1. Barros D, Ortega F. Metilfenidato e aprimoramento cognitivo farmacológico: representações sociais de universitários.Saúde e Sociedade.2011 jul;20:50-362.

2. Moreira SDNT, Silva CAN,Tertulino FDF, Tertulino FMDF, Vilar MJP,Azevedo GDD.Processo de significação de estudantes do curso de medicina diante da escolha profissional e das experiências vividas no cotidiano acadêmico. Rev Bras Educ Med.2006.30(2):14-19.

3. Arrais PSD, Coelho HLL, Batista MDCD, Carvalho ML, Righi RE, Arnau JM.Perfil da automedicação no Brasil. Revista de Saúde Pública..1997.31: 71-77.

4. Castro HC, Aguiar MLP, Geraldo RB, Freitas CC, Alcoforado LF, Santos DO, Toledo I.Automedicação: entendemos o risco. Infarma.2006. 18(9/10):17-20.

5. Naves JDOS, Castro LLCD,Carvalho CMSD,Merchán-Hamann E Automedicação: uma abordagem qualitativa de suas motivações.Ciência \& Saúde Coletiva.2010.15:1751-1762.

6. Carneiro SG,Prado,AST, de Jesus Araujo EC, Moura HC, Strapasson JF, Rabelo NF, Ribeiro TT. O uso não prescrito de metilfenidato entre acadêmicos de Medicina. Cadernos UniFOA.2013.8(1 (Esp.)):53-59.

7. Cruz TC,Barreto Junior EPDS,Gama MLM, Maia LCDM,Melo Filho MJXD,Manganotti Neto O,Coutinho DM.Uso não-prescrito de metilfenidato entre estudantes de medicina da Universidade Federal da Bahia.Gazeta Médica da Bahia.2011.81(1).Jun:3-6

8. Pastura G,Mattos P. Efeitos colaterais do metilfenidato. Archives of Clinical Psychiatry.2004.31(2):100-104.

9. Eslami AA,Jalilian F,Ataee M,Alavijeh MM,Mahboubi M,Afsar A,Aghaei A.Intention and willingness in understanding Ritalin misuse among Iranian medical college students: a cross-sectional study. Glob J Health Sci.2014 nov.6(6).43-53.

10. Da Silva ACP,Luzio CA,dos Santos KYP,Yasui S,Dionísio GH.A explosão do consumo de Ritalina.Revista de Psicologia da UNESP.2012.11(2):44-57. 
11. Moura MHD.As consequências do uso prolongado e não terapêutico do metilfenidato [monografia].Ariquemes:Faculdade de educação e meio ambiente.2017

12. Rosenberg MD,Zhang S,Hsu WT,Scheinost D,Finn ES,Shen X et al.Methylphenidate modulates functional network connectivity to enhance attention. $J$ Neuroscience.2016.Set.36(37):9547-9557.

13. Manza P,Hu S, Ide JS,Farr OM,Zhang S,Leung HC et al.The effects of methylphenidate on cerebral responses to conflict anticipation and unsigned prediction error in a stop-signal task.J Psychopharmacol.2016.Mar.30(3):283-293.

14. Ernst M,Lago T,Davis A,Grillon C.The effects of methylphenidate and propranolol on the interplay between induced-anxiety and working memory.Psychopharmacology.2016.Out.233(19-20):3565-3574.

15. Westover AN,Nakonezny PA,Adinoff B,Brown ES,Halm EA.Impact of Stimulant Medication Use on Heart Rate and Systolic Blood Pressure During Submaximal Exercise Treadmill Testing in Adolescents.J Child Adolesc Psychopharmacol.2016.Dez.26(10):889-899. 